say $0 \leqq \lambda \leqq 1$. Let $q_{i}=\varphi_{i}(\lambda), p_{i}=\psi_{i}(\lambda),(i=1,2, \cdots, n)$, where $\varphi_{i}, \psi_{i}$ have continuous derivatives on $\Lambda$ and $\left(\varphi_{i}, \psi_{i}\right)$ traces out a simple closed curve $c_{i}$ as $\lambda$ traverses $\Lambda$. This induces the relationships $Q_{i}=\Phi_{i}(\lambda), P_{i}=\Psi_{i}(\lambda)$. Because the transformation is canonical, $\left(\Phi_{i}(\lambda), \Psi_{i}(\lambda)\right)$ also traces out a simple closed curve $C_{i}$ in the $\left(Q_{i}, P_{i}\right)$ plane as $\lambda$ traverses $\Lambda$, and

$$
\begin{aligned}
\sum_{i=1}^{n}\left[\oint_{C_{i}} P_{i} d Q_{i}-\oint_{c_{i}} p_{i} d q_{i}\right] & =\sum_{i=1}^{n}\left[\int_{0}^{1} \Psi_{i}(\lambda) d \Phi_{i}(\lambda)-\int_{0}^{1} \psi_{i}(\lambda) d \varphi_{i}(\lambda)\right] \\
& =\int_{0}^{1} \cdot d H(q(\lambda), p(\lambda))=0 .
\end{aligned}
$$

Thus, correctly,

$$
\sum_{i=1}^{n} \oint_{c_{i}} P_{i} d Q_{i}=\sum_{i=1}^{n} \oint_{c_{i}} p_{i} d q_{i}
$$

Now the mistake is made of using Stokes' theorem $\oiint_{C} x d y=\iint_{A} d x d y$ to complete the "proof". But for this formula to hold, $C$ must be positively oriented. Now in the case at hand $C_{i}$ and $c_{i}$ have the orientations which are given to them by the mapping from $\Lambda$, and these are not necessarily all the same (examine the Example). We could use Stokes' theorem in the form

$$
\iint_{i i} d q_{i} d p_{i}=(-1)^{\sigma_{i}} \oint_{c_{i}} p_{i} d q_{i} \quad \text { and } \quad \iint_{S_{i}} d Q_{i} d P_{i}=(-1)^{\rho_{i}} \oint_{c_{i}} P_{i} d Q_{i},
$$

where $\sigma_{i}$ and $\rho_{i}$ are 0 or 1 according as $c_{i}$ or $C_{i}$ respectively is traversed negatively or positively as $\lambda$ traverses $\Lambda$. Thus a correct formulation is

$$
\sum_{i=1}^{n}(-1)^{\sigma_{i}} \iint_{i} d q_{i} d p_{i}=\sum_{i=1}^{n}(-1)^{\rho_{i}} \iint_{S_{i}} d Q_{i} d P_{i} .
$$

This subject, as well as other topics in mathematical physics, can be presented in a more unified and elegant fashion through the use of Cartan's exterior differential calculus. This the writer hopes to do in a later paper.

\title{
ON A GENERALIZATION OF SYNGE'S CRITERION FOR SUFFICIENT STABILITY OF PLANE PARALLEL FLOWS*
}

\section{BY S. I. PAI (University of Maryland)}

In Ref. [1], Synge derived sufficient conditions for the stability of plane Couette flow and plane Poiseuille flow of incompressible fluid. In the present note, the conditions are generalized so that the problem of velocity profiles with point of inflection and with either finite or infinite boundary condition or both may be included.

* Received August 10, 1953. This note was written when the author visited the Aeronautical Engineering Department of the Pennsylvania State College during August of 1953, supported by ONR Contract Nonr 656(01). 
The Orr-Sommerfeld equation [2] for stability of two-dimensional parallel flow of incompressible fluid may be written as follows:

$$
i \alpha R\left(w L-w^{\prime \prime}\right) \phi-i \alpha c R L \phi=L L \phi,
$$

where $L=d^{2} / d y^{2}-\alpha^{2}, x$ is the coordinate in the direction of the flow; $y$, the coordinate perpendicular to $x$; $t$, the time; $w=w(y)$, the velocity profile of steady flow; $\phi(y) \exp \{i \alpha(x-c t)\}$, the disturbance stream function; $c$, the disturbance phase velocity which may be complex, i.e. $c=c_{r}+i c_{i} ; \alpha$ the disturbance wave number which is chosen positive, and $R$, the Reynolds number of steady flow.

If the boundaries (finite or infinite) of the flow field are at $y_{1}$ and $y_{2}$, the boundary conditions on the disturbance amplitude function are given by

$$
\begin{aligned}
\phi\left(y_{1}\right) & =0, \quad \phi\left(y_{2}\right)=0, \\
\phi^{\prime}\left(y_{1}\right) & =0, \quad \phi^{\prime}\left(y_{2}\right)=0,
\end{aligned}
$$

where primes denote differentiation with respect to $y$.

Multiplying (1) by $\phi_{c} d y$ and integrating for the range $\left(y_{1}, y_{2}\right)$ we obtain

$$
i \alpha c R\left(I_{1}^{2}+\alpha^{2} I_{0}^{2}\right)=i \alpha R Q+\left(I_{2}^{2}+2 \alpha^{2} I_{1}^{2}+\alpha^{4} I_{0}^{2}\right)
$$

where

$$
\begin{gathered}
I_{0}^{2}=\int_{y_{1}}^{y_{2}} \phi \phi_{c} d y, \quad I_{1}^{2}=\int_{y_{1}}^{y_{2}} \phi^{\prime} \phi_{c}^{\prime} d y, \quad I_{2}^{2}=\int_{y_{1}}^{y_{2}} \phi^{\prime \prime} \phi_{c}^{\prime \prime} d y, \\
Q=\int_{y_{2}}^{y_{2}}\left[w \phi^{\prime} \phi_{c}^{\prime}+\left(\alpha^{2} w+w^{\prime \prime}\right) \phi \phi_{c}\right] d y+\int_{y_{1}}^{y_{2}} w^{\prime} \phi^{\prime} \phi_{c} d y,
\end{gathered}
$$

and the subscript $c$ indicates the conjugate complex quantity. The values of all the above integrals are finite for finite or infinite boundaries (see [3]).

Taking the imaginary part of (3) and simplifying, we have

$$
\int_{y_{1}}^{y_{2}}\left(w-c_{r}\right) \phi^{\prime} \phi_{c}^{\prime} d y+\alpha^{2} \int_{y_{1}}^{y_{2}}\left[\left(w-c_{r}\right)+\frac{w^{\prime \prime}}{2 \alpha^{2}}\right] \phi \phi_{c} d y=0 .
$$

If $c_{r}<0$, and $w$ is assumed positive, so that the first integral is positive, the second integral must be negative somewhere in the range $\left(y_{1}, y_{2}\right)$; thus we have

$$
c_{r}>\frac{w_{\min }^{\prime \prime}}{2 \alpha^{2}}+w_{\min },
$$

where $w_{\min }$ is a negative quantity.

In the case of $c_{r}>0$, if $c_{r} \geq w_{\max }$, the first integral is negative; the second integral must then be positive somewhere in the range $\left(y_{1}, y_{2}\right)$. Thus we have

$$
c_{r}<w_{\max }+\frac{w_{\max }^{\prime \prime}}{2 \alpha^{2}}
$$

where $w_{\max }^{\prime \prime}$ is a positive quantity. The value of $c$, must therefore lie in the following range:

$$
\frac{w_{\min }^{\prime \prime}}{2 \alpha^{2}}+w_{\min }<c_{r}<\frac{w_{\max }^{\prime \prime}}{2 \alpha^{2}}+w_{\max } .
$$


For plane Couette flow $w^{\prime \prime}=0$, we have

$$
w_{\min }<c_{r}<w_{\max } \text {. }
$$

For plane Poiseuille flow $w^{\prime \prime}=$ constant (negative quantity), $w_{\min }=0$ the sufficient conditions become

$$
\frac{w_{\min }^{\prime \prime}}{2 \alpha^{2}}<c_{r}<w_{\max } .
$$

Equations (6a) and (6b) have been found by Synge [1]. For flow without inflection point, the velocity of propagation of disturbances is either within a certain range (6a) or bounded on one side (6b). But for flow with inflection point, there is no bound for the velocity of propagation of disturbance (6).

Equation (6), however, does not tell us anything directly about stability. For the discussion of stability, we take the real part of (3); we have

$$
-2 \alpha c_{i} R\left(I_{1}^{2}+\alpha^{2} I_{2}^{2}\right)=i \alpha R I_{m}\left(Q-Q_{c}\right)+2\left(I_{2}^{2}+2 \alpha^{2} I_{1}^{2}+\alpha^{4} I_{0}^{2}\right)
$$

where

$$
I_{m}\left(Q-Q_{c}\right)=I_{m}\left[2 \int_{y_{1}}^{y_{2}} w^{\prime} \phi^{\prime} \phi_{c} d y\right]
$$

It is easily seen that

$$
\left|I_{m}\left(Q-Q_{c}\right)\right|<2 q I_{0} I_{1}
$$

where $q=\left|w^{\prime}\right|_{\max }$.

For any real constant $a$ and $b$, we have

$$
M=\int_{\nu_{1}}^{y_{3}}\left(\phi+a w^{\prime} \phi^{\prime}+b \phi^{\prime \prime}\right)\left(\phi_{c}+a w^{\prime} \phi_{c}^{\prime}+b \phi_{c}^{\prime \prime}\right) d y>0
$$

or

$$
\begin{aligned}
M=I_{0}^{2}+a^{2} \int_{y_{1}}^{y_{2}} w^{\prime 2} \phi^{\prime} \phi_{c}^{\prime} d y+b^{2} I_{2}^{2}-a \int_{y_{1}}^{\nu_{2}} & w^{\prime \prime} \phi \phi_{c} d y, \\
& -2 b I_{1}^{2}-a b \int_{y_{1}}^{\nu_{2}} w^{\prime \prime} \phi^{\prime} \phi_{c}^{\prime} d y>0 .
\end{aligned}
$$

Hence, if $a$ and $b$ are any positive numbers,

$$
b^{2} I_{2}^{2}>I_{1}^{2}\left(2 b+a b p-a^{2} q^{2}\right)+I_{0}^{2}(a p-1)
$$

where

$$
p=\left|w^{\prime \prime}\right|_{\max } .
$$

Substituting (9) and (12) into (7) we have

$$
\begin{aligned}
\alpha c_{i} R b^{2}\left(I_{1}^{2}+\alpha^{2} I_{0}^{2}\right)<b^{2} \alpha R q I_{0} I_{1}-I_{1}^{2}\left(2 b+a b p-a^{2} q^{2}+\right. & \left.2 \alpha^{2} b^{2}\right) \\
& -I_{0}^{2}\left(a p-1+b^{2} \alpha^{4}\right) .
\end{aligned}
$$

Therefore the stability condition $c_{\boldsymbol{i}}<0$ is satisfied provided

$$
\left(b^{2} \alpha R q\right)^{2}<4\left(2 b+a b p-a^{2} q^{2}+2 \alpha^{2} b^{2}\right)\left(a p-1+b^{2} \alpha^{4}\right),
$$


where $a$ and $b$ are any positive real constants satisfying

$$
2 b+a b p-a^{2} q^{2}+2 \alpha^{2} b^{2}>0, \quad a p-1+b^{2} \alpha^{4}>0 .
$$

Lessen's criterion [3] corresponds to $p=0, q=1$ of (16).

We may choose some values for $a$ and $b$ to get a criterion for jet flow. If we put

$$
a=K / \alpha p, \quad b=K q^{2} / \alpha p^{2},
$$

where $K$ is an arbitrary constant such that $K \geq 1$ and $K^{2} q^{4} / p^{4} \geq 1$, Eq. (16) is satisfied for all $\alpha$ and (15) becomes

$$
(R q)^{2}<8\left[\alpha^{4}+\frac{p^{2}}{K q^{2}} \alpha^{3}-\frac{p^{4}}{K^{2} q^{4}} \alpha^{2}+\left(1-\frac{p^{2}}{K^{2} q^{2}}\right) \frac{p^{4}}{K q^{4}} \alpha+\frac{p^{6}}{K^{2} q^{6}}\right]
$$

Equation (18) seems to be the criterion which is suitable for jet type flow. As $\alpha$ tends to be zero, the flow will be stable for a finite Reynolds number.

\section{REFERENCES}

(1) J. L. Synge, Hydrodynamical stability, Semi-centennial Pub. Am. Math. Soc. Vol. 2, New York 1938, pp. 227-269.

(2) C. C. Lin, On the stability of two-dimensional parallel flow: Part I-General theory, Q. Appl. Math. 3, 117-142 (1945).

(3) M. Lessen, Note on a sufficient condition for the stability of general, plane parallel flows, Q. Appl. Math. 10, 184-186 (1952).

\section{A note on my paper}

\section{A FORMULA FOR AN INTEGRAL OCCURRING IN THE THEORY OF LINEAR SERVOMECHANISMS AND CONTROL-SYSTEMS}

Quarterly of Applied Mathematics, X, 205-213 (1952)

\section{BY HANS BÜCKNER}

Replace $D_{n}^{-1}$ by $D_{n-1}^{-1}$ in formula $\left(36^{\prime}\right)$, replace $a_{1}^{2}$ by $a_{1}^{3}$ in formula (44), replace $2 Y$ by $Y$ in formula (45). Formula (46) is correct and corresponds to corrected formula (44).

The author wishes to thank Mr. Kenneth Geohegan and Mr. Edwinn Kinnen for their check of formulae $\left(36^{\prime}\right)$ and (44).

\section{BOOK REVIEWS}

50-100 Binomial tables. By Harry G. Romig. John Wiley \& Sons, Inc., New York, and Chapman \& Hall, Ltd., London, 1953. xxvii + 172 pp. \$4.00.

The value of the individual terms and the sum of the first $x$ terms of $[P+(1-P)]^{n}$ are given for $n$ in the range 50 to 100 in steps of 5 and $p$ in the range 0.01 to 0.50 in steps of 0.01 to six decimal places although the last place is doubtful. The introduction, written in a clear and concise manner, describes the use of the tables, its application to quality control and its relation to the ratio of the incomplete $\beta$-function to the complete $\beta$-function. The tabulated data are easy to read but the nature of the entries prevents a more uniform tabulation.

S. L. LevY 